\title{
Microtopographic Analysis Of Part-Through Crack Growth In Allow 304L Plate-Type Tension Speciments
}

Wilson R. Lloyd

Eric D. Steffler

John H. Jackson

April 2003

Idaho National Engineering and Environmental Laboratory Bechtel BWXT Idaho, LLC 


\title{
Microtopographic Analysis of Part-Through Crack Growth In Alloy 304L Plate-Type Tension Specimens
}

\author{
Wilson R. Lloyd \\ Eric D. Steffler \\ John H. Jackson
}

April 2003

\section{Idaho National Engineering and Environmental Laboratory Materials Department Idaho Falls, Idaho 83415}

Prepared for the

U.S. Department of Energy Office of Science Under DOE Idaho Operations Office

Contract DE-AC07-99ID13727 


\title{
Microtopographic Analysis of Part-Through Crack Growth in Alloy 304L Plate-Type Tension Specimens
}

\author{
W. R. Lloyd \\ E. D. Steffler \\ J. H. Jackson \\ Idaho National Engineering and Environmental Laboratory \\ Materials Dept. \\ PO Box 1625 \\ Idaho Falls, ID 83415-2218
}

\begin{abstract}
The Idaho National Engineering and Environmental Laboratory (INEEL) used their microtopography analysis method to examine the fracture process in two Type 304 stainless steel, part-through crack, plate-type specimens. The two specimens had different initial defect geometries - one being nearly semicircular and moderately deep, the other being longer and shallower. The microtopographic analysis allowed determination of parameters such as: the crack tip opening displacement at initiation; the crack tip opening angle during ductile tearing; the crack mouth opening at through-thickness penetration; and, the incremental crack front profiles throughout the crack growth process. In essence, these data provide a nearly complete description of the entire ductile fracture process for the two cases examined.

We describe the microtopographic analysis procedure as it was applied to these two specimens. Crack growth profiles predicted by the microtopography analysis are compared with those shown by heat tinting of the actual fracture surface, showing excellent agreement. Several areas of ductile crack growth theory relevant to the microtopographic method of analysis are discussed, including possible effects on the accuracy of the analyses. The accuracy of the resultant data is reviewed, and found acceptable or better. Areas for additional development of the microtopography method to improve accuracy in three-dimensional ductile fracture analysis are identified.
\end{abstract}




\section{BACKGROUND}

Engineered structures with thin cross-sections, made of tough, ductile materials are often designed, and sometimes analyzed, using basic limit-load analyses. Inspections of these structures after they have been in service for some time can reveal cracks. If the applied "failure" definition is one of plastic collapse, the limit-load analysis will often be satisfactory. However, in the case of pipes, tanks, or pressure vessels, where leakage may be the "failure," there is no currently accepted method of reliable prediction. It is in this area of fracture mechanics analysis that knowledge of the crack growth process is limited, and methods to predict the point of penetration during overload conditions is non-existent. Many factors interact; including the material's sensitivity to constraint near the crack tip, material hardening, ductile tearing resistance, and the three-dimensional geometry of the growing part-through surface crack (PTC). This paper presents a preliminary assessment of the capabilities offered by the Idaho National Engineering and Environmental Laboratory's Microtopography System $[1]$ in assessing the ductile fracture process in PTC geometries.

\section{Microtopography Analysis}

The three-dimensional nature of the surface crack geometry, combined with the non-linear ductile fracture process causes the assessment of the ductile fracture process in these cases to be quite complex. The capabilities of the microtopography ductile fracture analysis method discussed in this report provide key information to the modeling effort and overall understanding of these fracture processes. The method provides a full internal representation of the complete ductile fracture process, from initial crack tip blunting through extensive ductile tearing.

The "fracture process state," a unique descriptor of any instant during the ductile fracture process, can be related to a single-valued test parameter such as the crack mouth opening displacement (CMOD). Any observed value of CMOD is representative of some unique state of the fracture process in the specimen.

Microtopography analysis allows extraction of key fracture process parameters, including:

- crack border shapes (growth increments) as a function of applied CMOD (or force). (These shapes are the locus of (x,y) crack tip positions at a given process state.)

- crack opening profiles, at any location/section, at any process state. (These are section views normal to the nominal fracture plane, and parallel to the nominal growth direction. They provide various information about local crack state, out-of-plane zig-zagging, void growth ahead of the tip position, etc.)

- crack tip opening displacements at initiation $\left(\mathrm{CTOD}_{\mathrm{i}}\right)$, extracted from the opening profile view. (initiation, the transition from blunting to tearing, occurs progressively, and possibly simultaneously at different locations, along the instantaneous crack border as a function of fracture process state. The first noted initiation, typically the lowest value of $C^{2} \mathrm{CD}_{\mathrm{i}}$ observed, is usually designated the "critical CTOD" for a particular material and initial crack geometry combination.)

- crack tip opening angles (CTOA), correlated to fracture surface position, process state, and "local" crack extension.

Related to CTOA, and the fracture process characterization in general, the "local" crack extension measurement must be defined in some consistent manner. Crack growth in these PTC specimens is 
never really "self similar," i.e. the border shape is continuously changing, leading to curved growth trajectories. This is why the issue of crack extension measurement needs to be addressed. A possible definition would measure the crack growth as an arc length along a crack growth trajectory curve between the instantaneous position and the corresponding position on the initial defect perimeter. Such growth trajectory paths could be mapped from the microtopography data.

These parameters and/or data are all available as an essentially continuous function of fracture process state, at any location on the fracture surface that exists at that state. For the specimens being tested, these fracture process states can be correlated to externally-measurable parameters such as crack mouth opening, and subsequently to applied stress and other possible test measurements. The following description and analysis results demonstrate the general capabilities of the microtopography methodology at its present state of development.

Two areas within the microtopography analysis method do require further development. One is the issue of plastic flow behind the crack tip region (intersecting crack flank surfaces) early in the fracture process. The other concerns the transform of $(\mathrm{x}, \mathrm{y})$ coordinate locations of material points on the fracture surface as the fracture process progresses. Both of these issues have potential effects on the interpretation and or accuracy of microtopography results, and are discussed in some detail later in this paper. The following results show that many of the process parameters needed to characterize the ductile fracture process in PTC-type geometries are accurately measured without addressing these concerns. We also describe experimental methods to develop the additional microtopography analysis capabilities needed to completely characterize all aspects of the ductile fracture process for any defect geometry.

\section{Specimen Description and Original Testing}

We selected two representative PTC-type plate specimens with different PTC geometries for the investigation. These specimens were tested by INEEL about 20 years ago, and had been retained for reference following the initial tests. The specimen material is Alloy $304 \mathrm{~L}$ stainless steel - commonly used for piping and pressure vessels in chemical and power generation facilities.

The plate specimens $-\# 7$ and \#8 - were of the "dog-bone" shape. The test sections of the plate specimens were $6.35 \mathrm{~mm}$ thick and $152 \mathrm{~mm}$ wide. The ends were $290 \mathrm{~mm}$ wide and $12.7 \mathrm{~mm}$ thick. Both specimens had thin, part-through, semielliptical notches inserted in them via electric discharge machining (EDM). Specimen \#7 had a notch of approximately semicircular form, with a depth of about $75 \%$ of the plate thickness. Specimen \#8 had a shallower semielliptical notch. The depth appears to have been about $15 \%$ of plate thickness, and the elliptical aspect ratio, $c / a,{ }^{1}$ was about 4 .

The end sections were reinforced with hard carbon steel plates $12.7 \mathrm{~mm}$ thick. These reinforcing plates were attached to either face of the end section with two 3/4-inch bolts and two 1-inch bolts. The specimens were loaded in tension with clevises and a 2.25-inch pin passing through the specimen and both reinforcing plates.

The specimens were tested in displacement control mode at a constant rate (rate unknown, but relatively slow). The test continued for both specimens until just after the defects were observed to penetrate the plate thickness. The point of penetration was noted in the test record. The test procedure was continued until the applied force was observed to begin decreasing. For both specimens, the

\footnotetext{
${ }^{1} c$ is the half-width of the crack at the front face. $a$ is the center depth, the maximum for a true semi-elliptical crack.
} 
point of maximum applied force was well past the point where the starter notch initiated a crack and grew through the specimen thickness. The specimens were unloaded and removed from the test machine and heat tinted to mark the extent of crack extension in the specimen interior. The remaining ligaments at either side of the final crack tip position were then sawed into, to within about $3-5 \mathrm{~mm}$ of the apparent crack tip. The specimens were returned to the test machine and loaded until the two halves separated across the small remaining ligament. The heat tinting then revealed the extent of crack growth that had occurred during the initial test loading, as shown in Figure 1 and Figure 2.

At the test termination point, specimen \#7 had crack extension across the plate width to a total length of about $45 \mathrm{~mm}$ (see Figure 1). The crack in specimen \#8 had penetrated the thickness, and had extended such that crack length along the back surface was almost the same as the original front surface length (see Figure 2). The original front surface notch tips were either still in a blunting mode, or were just transitioning to tearing.

Notice the drastic tunneling of the crack in Specimen \#7. The onset of tunneling is also apparent in Specimen \#8. Although these figures do not have scales, the final thickness in the lateral crack growth area of Specimen $\# 7$ is about $3 \mathrm{~mm}-1 / 2$ of the original plate thickness. The scale of both pictures is about the same. The COD gage knife edge, visible on the crack mouth side of both specimens, is $6.5 \mathrm{~mm}$ wide on \#7, and $6.2 \mathrm{~mm}$ wide on \#8. The "A" and "B" designators stated in the captions were assigned to keep track of the data from the two halves of the specimens during the microtopographic analysis procedures. It is obvious that extreme plastic deformation has occurred during crack growth in both cases. We applied the microtopography method of crack growth analysis to both of these specimens with very promising initial results.

\section{Microtopography Data Collection and Analysis Procedure}

Lloyd provides details of the microtopography method of analysis in two papers [1,2]. The following sections provide an overview of the application to the PTC specimens examined in this work.

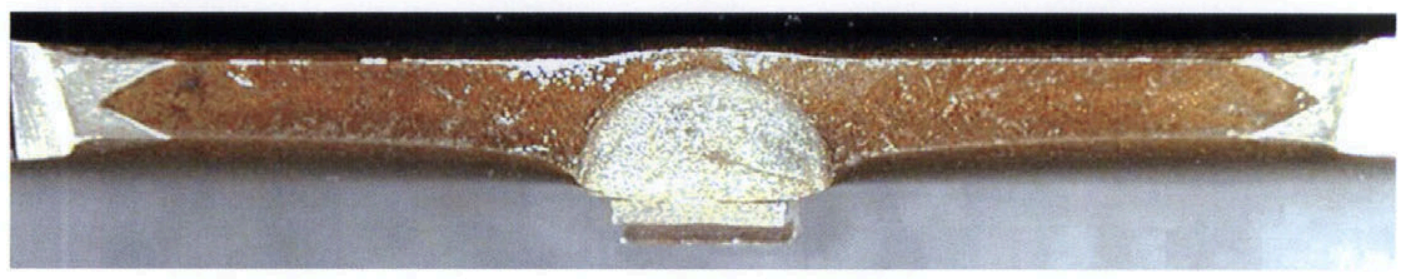

Figure 1. Specimen \#7 - fracture surface of "B" side.

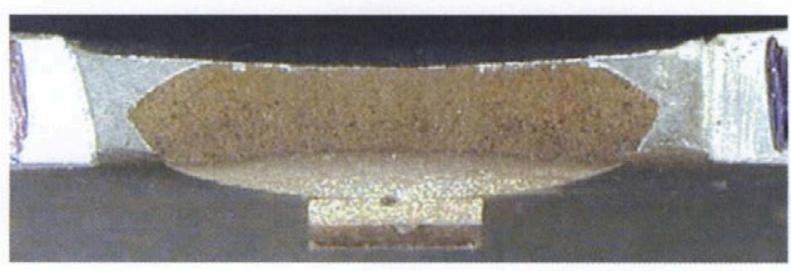

Figure 2. Specimen \#8 - fracture surface of "A" side. 


\section{Collecting the Raw Height Data}

The INEEL microtopography system collected high-resolution height data from the fracture surfaces of these specimens. Referring to Figure 1 and Figure 2, the $\mathrm{X}$ coordinate is to the right and the $\mathrm{Y}$ coordinate is up, as viewed on the page. The origin is located approximately at the $\mathrm{X}$ center of the crack mouth. Microtopography makes point height measurements $(z)$ at prescribed $(x, y)$ coordinate locations. Data were collected on a regular rectangular grid for both of these specimens. For \#7, the $\mathrm{X}$ spacing was $0.2 \mathrm{~mm}$, and the $\mathrm{Y}$ spacing was $0.1 \mathrm{~mm}$. For \#8, the spacing was $0.1 \mathrm{~mm}$ for both $\mathrm{X}$ and $\mathrm{Y}$. This results in 10,000 to 12,000 data points for each of the surfaces measured. Height measurement accuracy is the order of 3 to $5 \mu \mathrm{m}$ (resolution is sub- $\mu \mathrm{m}$ ), and the measurement range is about $30 \mathrm{~mm}$. This is far more than adequate for these specimens. The height range over the fracture surfaces of these specimens was about $3 \mathrm{~mm}$ for \#8, and about $7 \mathrm{~mm}$ for \#7. Figure 3 shows representative 3-D wireframe plots of the surface height data from the two specimens.

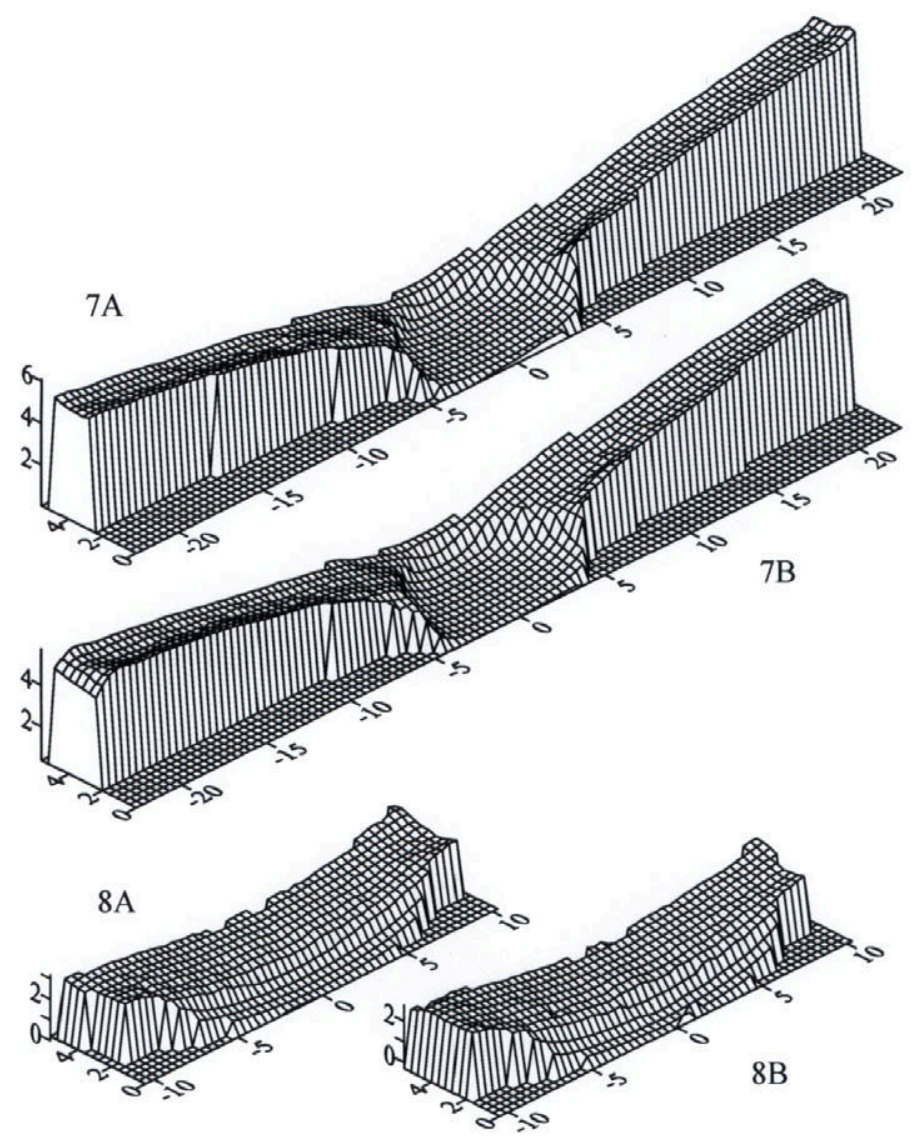

Figure 3. 3-D fracture surface heights as measured from the two specimens. There is approximately 10 times as much actual data as that represented by the wireframe grids. Scaling is proportional in $x: y: z$ (true representation of surface), dimensions are in mm. 


\section{Mapping the Crack Front Positions During the Fracture Process}

Data are collected at corresponding $(x, y)$ coordinate locations on the two surfaces. Careful alignment and matching of point heights at known states allows creation of a height difference (dz) map. The properly created height difference map, displayed as a dz contour plot, then represents instantaneous position of the growing crack border. This gives a continuous representation of the crack border location during the fracture process. Crack initiation locations, crack shape change, and the evolution and extent of crack tunneling all throughout the fracture process are revealed. Figure 4 and Figure 5 show such contour plots for the two specimens examined. For improved comprehension, the contours are superimposed on images of the actual fracture surface. Scaling is adjusted so all $(\mathrm{x}, \mathrm{y})$ locations correlate between the image and contour plot. Notice where specimen heat tinting was applied to mark an instantaneous crack tip location on both specimens, and how well the microtopography height difference contours match these known border locations. The accuracy of both the shape and instantaneous tip location is very good for both specimens.

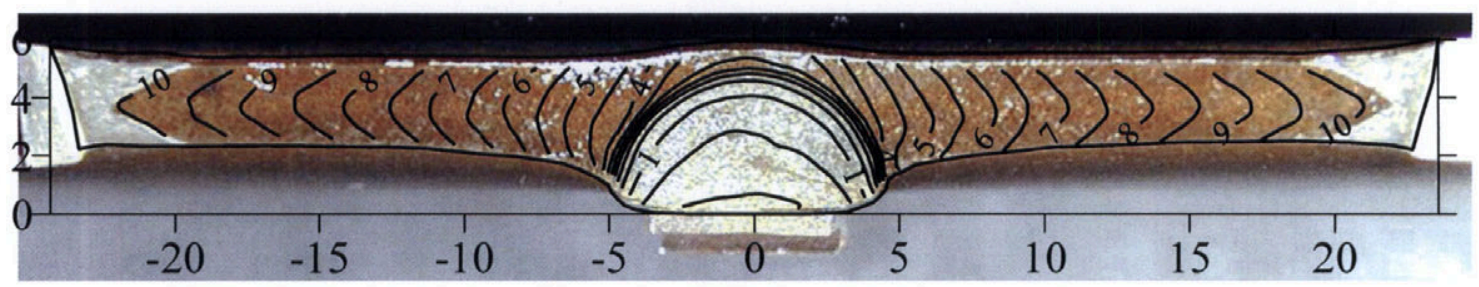

Figure 4. \#7 surface with opening contour overlay

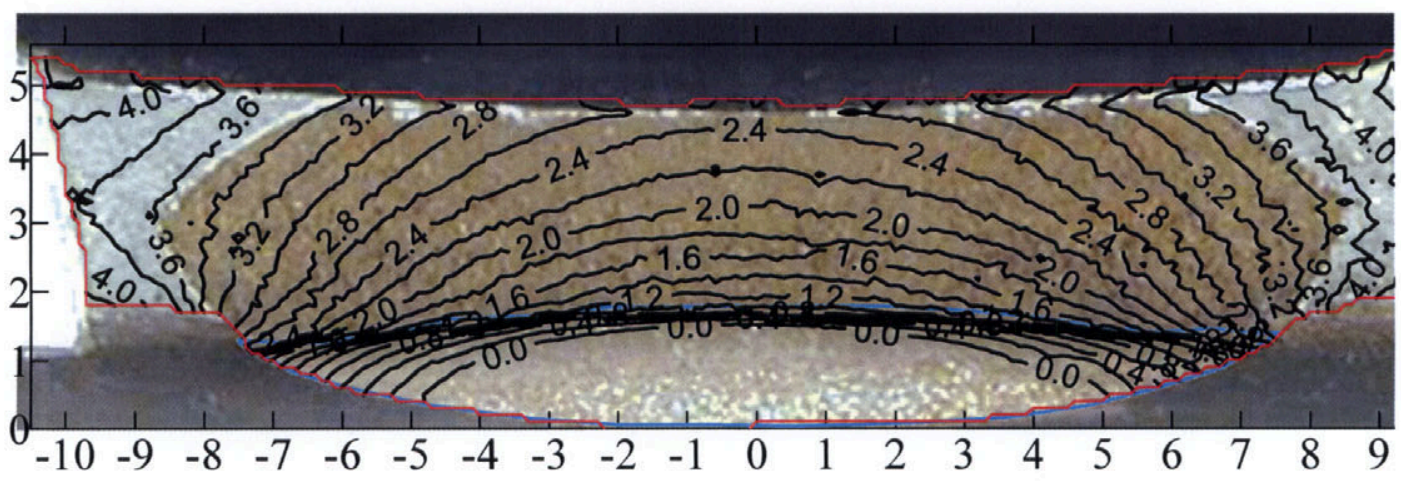

Figure 5. \#8 surface with opening contour overlay

\section{Inference of Fracture Process CMOD From Height Differences}

The magnitude of a given separation distance contour line(s) can be related to the crack mouth opening displacement when the crack border was at that position. Referring to Figure 4 (showing specimen \#7 fracture surface in plan view), and with the crack at the state where the specimen was heat tinted, we see that the CMOD would have been about $10.0 \mathrm{~mm}$. By comparing the relationship of the heat tint border to the nearby height contours, the estimated CMOD value seems to be reliable 
within a few tenths of a millimeter. An old photograph of the crack mouth at the end state was digitized and measured for a comparison point (see Figure 6). Note that this was done after the above analysis, and that this measured value was in no way used to alter or "fudge" the results. The CMOD measured from the photograph was $9.89 \mathrm{~mm}$, just a little over a $1 \%$ discrepancy.

Figure 7 shows the same comparison and correlation for specimen \#8. As can be seen, the level of correlation at the crack growth state marked by the heat tint has similar accuracy. There is a bit more mismatch in crack border shape near the intersection of the original crack (notch) ends and the front face surface. Figure 7 shows the front face of specimen \#8 at the end of the test. It is not surprising that the basic microtopography model does not precisely emulate crack growth in the presence of extreme multi-dimensional plasticity at these two locations. This particular issue will need to be addressed at some time, since assessment of these types of regions will eventually be required for a complete analysis.

The accuracy of the estimated crack border position versus instantaneous CMOD in these regions is probably still within $0.5 \mathrm{~mm}$ of the actual location in the $\mathrm{x}$-direction, based on microtopography-toheat tint border location comparisons. The comparison of the CMOD measured from the photograph with the microtopography CMOD is not as good as that for specimen \#7, but still acceptable. Note that the contour interval of 0 does not correspond to the outer, center position on the crack mouth. That value is about $-0.8 \mathrm{~mm}$, so the actual estimated CMOD at the border contour location nearest the heat tint contour is about $4.4 \mathrm{~mm}$. The measurement from the photograph results in value of $5.4 \mathrm{~mm}$. That is an error of about $-18 \%$ in the CMOD prediction by microtopographic analysis. Bear in mind that these microtopography analyses were from 20 -year old specimens, and some estimation had to be made concerning alignment and height correlation of the two surfaces. With proper care during testing, and appropriate specimen preparation, accuracy to less than 10\% CMOD error when correlating crack border position to fracture process CMOD is achievable.

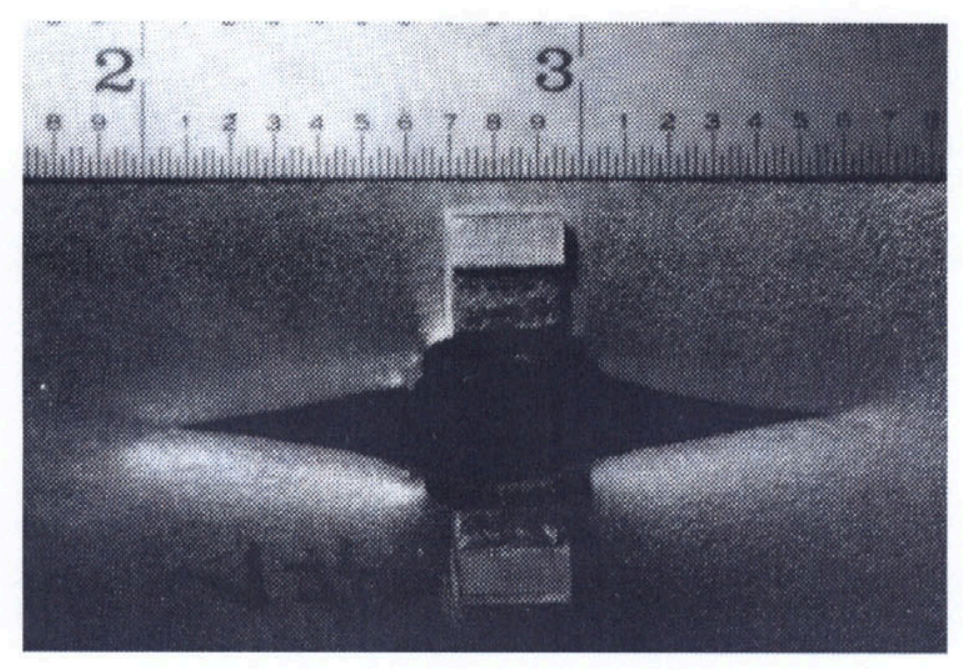

Figure 6. Front face of specimen \#7 at conclusion of loading. Heat tint on fracture surface shown in Figure 4 corresponds to this surface state. 


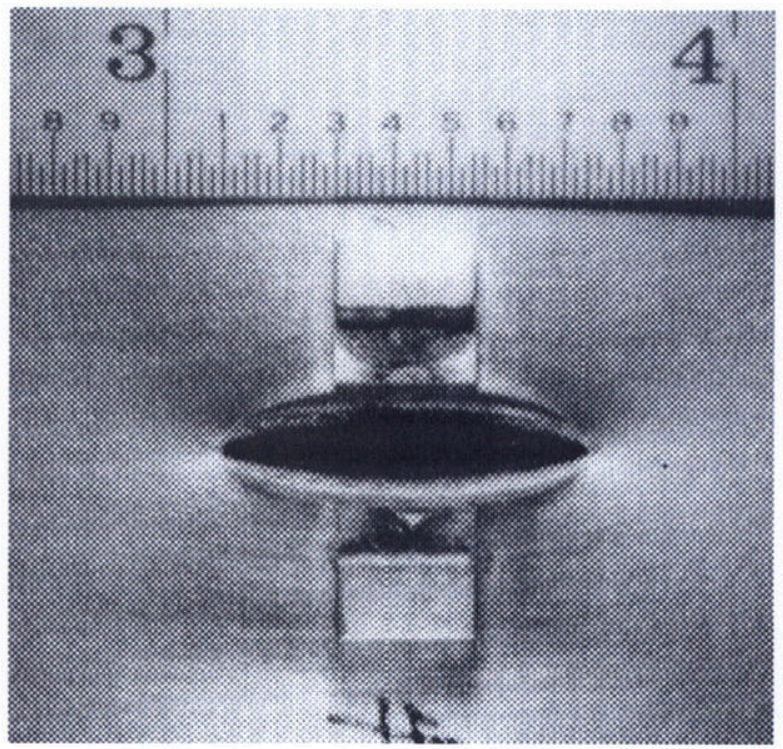

Figure 7. Front face of specimen \#8 at conclusion of loading. Heat tint on fracture surface shown in Figure 5 corresponds to this surface state.

\section{Transformation of the $(\mathrm{X}, \mathrm{Y})$ Coordinate System During the Fracture Process}

One issue that still needs to be addressed is that of the specimen distortion in the $(\mathrm{x}, \mathrm{y})$ plane as crack growth occurs. It is obvious that the originally flat front and back specimen faces are no longer planar in the vicinity of the nominal crack plane. The crack perimeter contours displayed in the Figures represent the state at the conclusion of testing. Positions of the crack border at earlier stages in the growth process are obviously inaccurate relative to $(x, y)$ coordinates at that instant in the process. However, there will still be point-for-point correlation between the upper and lower surfaces, even though the $(\mathrm{x}, \mathrm{y})$ coordinate system has undergone a transformation during crack growth. This implies that the separation data at a given final $(\mathrm{x}, \mathrm{y})$ coordinate is still correct. This logic indicates that the crack border shapes during early stages of crack growth are not accurately represented by the final state height contours.

An experimental approach to develop a coordinate transform model that can be used in the process model to correct the positional error has been devised. Specially instrumented tests on representative PTC specimens would be performed and out-of-plane surface deformation would be mapped and correlated to CMOD. These data could then be applied in an incremental "boundary value" sense to correct $(\mathrm{x}, \mathrm{y})$ coordinates across the fracture surface for each fracture process increment. It is presented and discussed in more detail in "Research Plans \& Requirements for Microtopographic Analysis."

\section{Extracting CTOA From the Height Map}

The next stage of the analysis procedure is to extract the CTOA at desired locations and for varying amounts of crack extension. It may have become apparent that the crack opening height difference, when compared to local position changes on the fracture surface, $\mathrm{dz} / \mathrm{ds}$, is directly equivalent to a local crack tip opening angle. The actual extraction is a somewhat tricky business, since the local direction of crack extension must be assessed. It seems that a reasonable first estimate can utilize the 
gradient operator to sense the local maximum slope direction, and to then determine that slope. This is not a perfect solution, especially in the case of severe tunneling (e.g. Specimen \#7 later stages of crack growth) or drastic localized crack shape/direction change. These problem areas are discussed later. However, the gradient method does provide a starting point for analysis, and it can be performed in an automated fashion using discrete numerical analysis techniques - a very desirable point to save analysis time.

Figure 8 and Figure 9 show contour plots of CTOA measured using this gradient analysis method in $(\mathrm{x}, \mathrm{y})$-space for the two specimens. This is a discrete, interpolated, point-to-point gradient analysis that is performed by the Golden Software ${ }^{\circledR}$ Surfer $®$ v7.0 (or later versions) 3-D graphing and data analysis software. Selecting menu item "Grid..Calculus..Derivative \& Integral Operations," and using the "Gradient Operator" option gives these results. Recall that the raw data was collected from the fracture surfaces at a spacing of $0.1 \mathrm{~mm}$, and there is some "noise" in the data. This combination of small "ds" and some noise in "dz" creates amplified "noise" in the gradient results. The data presented here were not smoothed, so the contours in areas of relative constant CTOA seem to be quite irregular at first inspection. Further analysis using sectioning and smoothing techniques will provide results more directly applicable for boundary conditions or comparison to continuum analysis methods, e.g. slip line analysis. In these two example specimens, there are two areas where the CTOA determined from the height difference gradient will certainly be suspect. One area is in the vicinity where the original crack/notch intersects the front surface, as discussed in Section 0, "Inference of Fracture Process CMOD From Height Differences." This will certainly be the area requiring the most development in microtopography analysis to derive accurate CTOA values. There are no estimates of potential error in this region, since there are no data available from independent measurements on which to base a comparison. As mentioned earlier, this region of the fracture surface should not be of

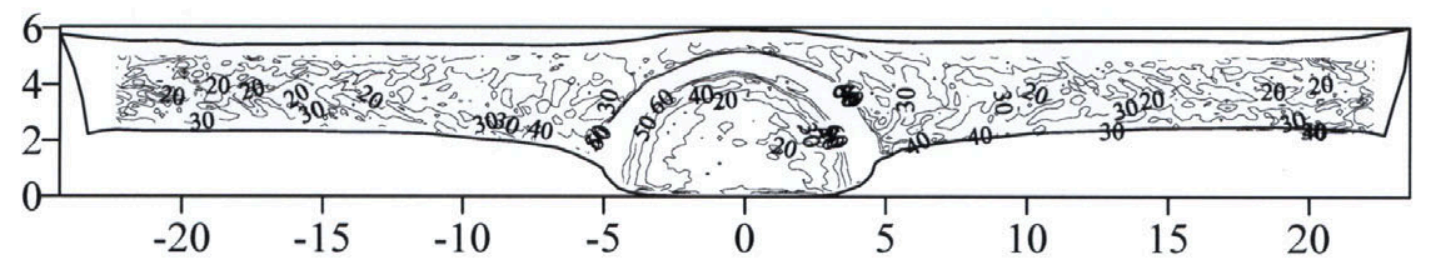

Figure 8. Specimen \#7 CTOA estimation based on gradient operator calculation

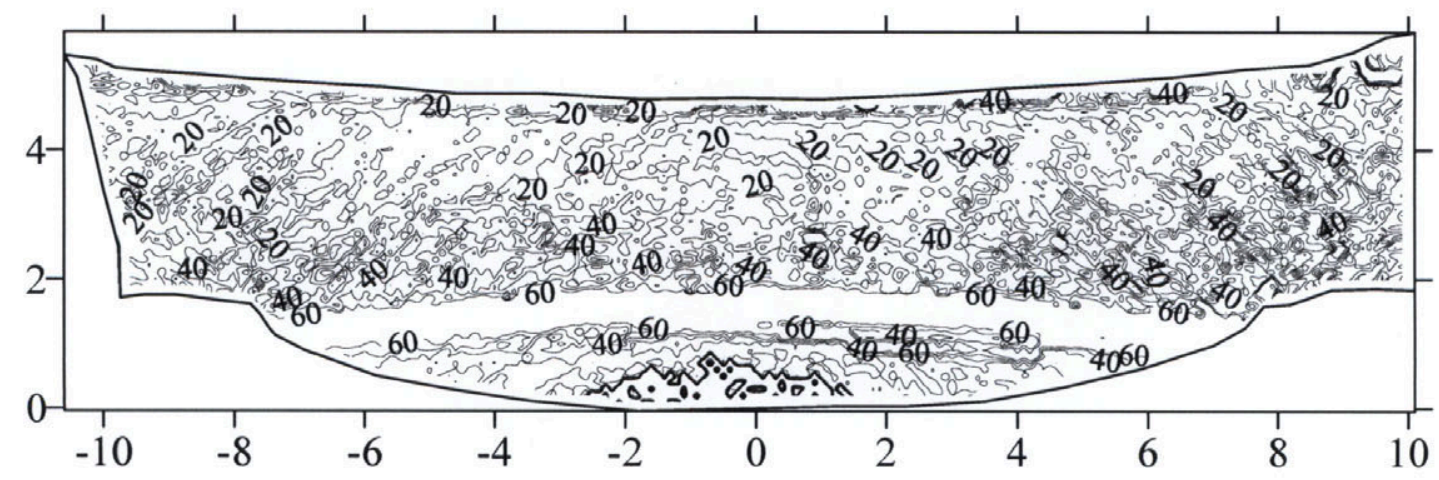

Figure 9. Specimen \#8 CTOA estimation based on gradient operator calculation 
direct importance until sometime in task 2 of the project. The other problem area is where there is substantial crack tunneling, as seen at the left and right portions of the fracture surface of Specimen \#7 (see Figure 4). However, this is not a significant problem area for analysis, since the local direction of crack extension is well established. Simply taking the directional derivative parallel to crack growth $-|\mathrm{d}(\mathrm{dz}) / \mathrm{dx}|$ in the case of $\# 7$ - will give the desired slope value in the appropriate direction. Figure 10 shows this result for Specimen \#7. Areas of high indicated CTOA associated with the stretch zone have been removed from the plot for clarity. Note the obvious error in the assessment where the crack is growing through the thickness direction - maximum initial crack depth, $\mathrm{x} \approx 0$. These bulk data operations are, however, useful for a general overview of CTOA trends in various regions of the specimen.

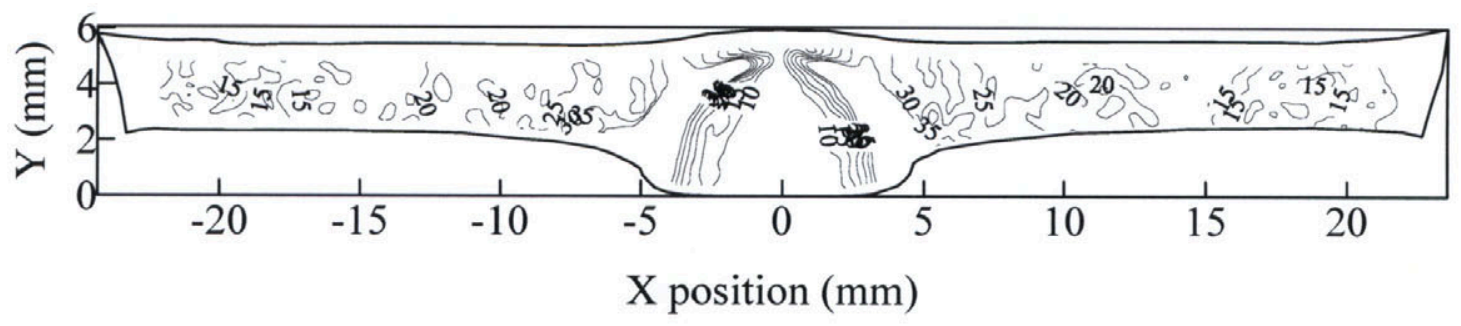

Figure 10. Specimen \#7 CTOA contours (deg) based on the absolute value of the $\mathrm{x}$-direction derivative $(\mathrm{d}(\mathrm{dz}) / \mathrm{dx})$.

Once specific regions are identified where detailed CTOA information is necessary, profile sections can be extracted on a trajectory parallel to the local crack growth (this could be a curving path, of course), and the CTOA can be determined using 2-D analysis. As an example, consider Figure 11 showing the height profiles of four sections taken parallel to the nominal crack growth direction (parallel to X, after crack had penetrated thickness and turned to strictly lateral growth across the specimen plate width). Several significant features of the fracture process in these regions can be deduced from this plot. An obvious one is the relatively symmetric growth as indicated by equivalent height difference values, dz, at corresponding + and -X locations. Secondly, the degree of tunneling can be assessed based upon the lateral shift required to overlay the centerline height difference profile with the near-back surfce height difference profile. In the case of Specimen \#7, this ranges from a little less, to a little more, than $1 \mathrm{~mm}$, with tunneling increasing with increasing crack extension (the offset increases). Also, notice the obvious negative curvature of the plots of height difference, indicating a decreasing CTOA with increasing crack extension. This is consistent with the fact that the 304SS material strain hardens a great deal. So, hardening of the remaining ligament was continually increasing with each increment of crack extension. Experience with CTOA assessment suggests a corresponding decrease of CTOA with increasing material strength. Crack tunneling was also observed to increase with increasing crack growth. Possible constraint increase at mid-thickness associated with increasing hardening may partially explain the increased tunneling effect.

As a further point of comparison, CTOA measured at the specimen outer surface can be compared to the near-surface values derived using microtopographic data. The available images were rather poor, being 20-year old PMTs (half-tones) at low magnification. The front (see Figure 6) and back surfaces of Specimen \#7, including both crack tips, all had CTOA measured at $17 \mathrm{deg}$ (within measurement accuracy capability). Specimen \#8 CTOA was assessed at the back surface only (see Figure 12), and measured 16 deg at both tips. The front surface (Figure 7) appears to be still in transition from blunting to tearing, so a meaningful CTOA could not be determined from that image. Given the image quality, and limited measuring accuracy, both of these surface angles compare well with the near surface CTOA values extracted using microtopography - about 13-15 deg in both cases. 


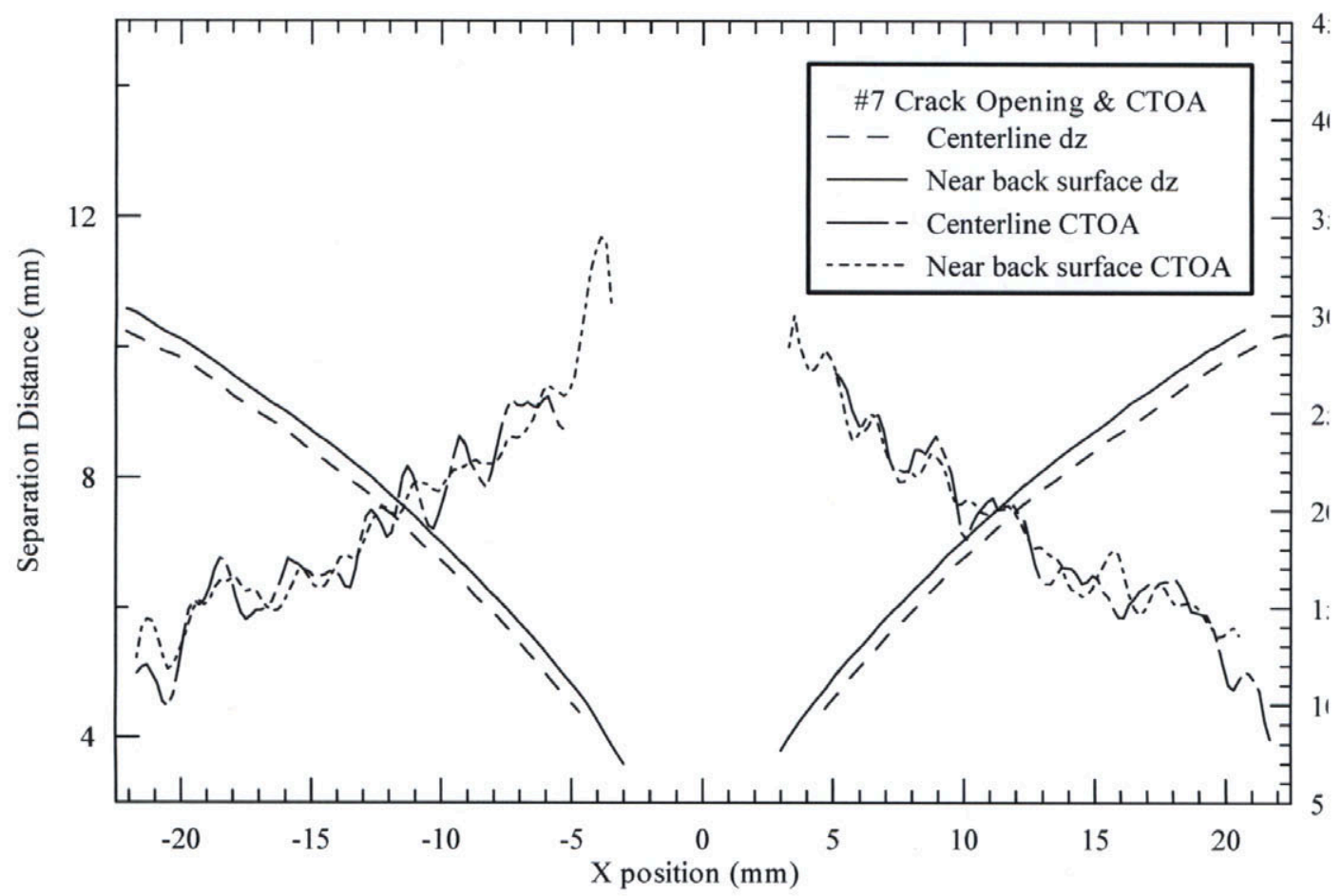

Figure 11. Surface height difference profiles for dominantly lateral crack growth in Specimen \#7.

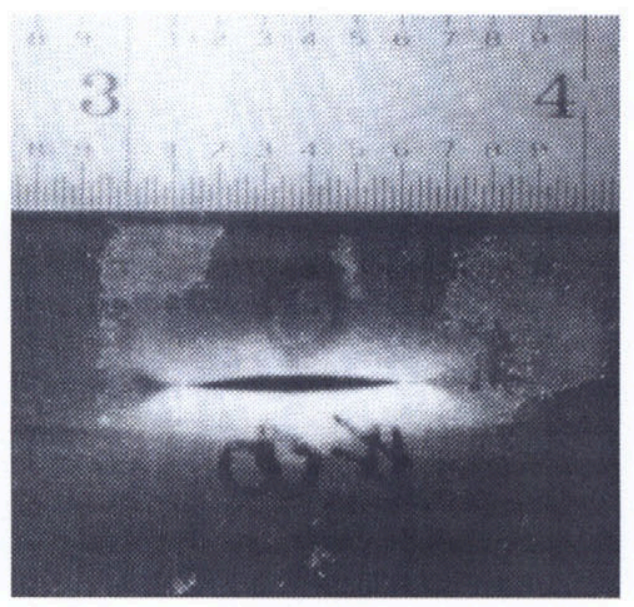

Figure 12. Back surface of Specimen \#8 at test conclusion (heat tint marking of fracture surface).

\section{Assessing Local Initiation CTOD Values}

The sectional height difference information can also be viewed in the form of actual, physical crack opening profiles. For the quasi-steady-state case depicted in Figure 11, these types of profiles provide 
visually interesting plots, but little additional information. However, this type of presentation is very valuable for examination of the early crack growth behavior. Recall that the initial notch/crack in specimen \#7 had a nearly semicircular profile with a radius of about $4.5 \mathrm{~mm}$. Actual surface height profiles were extracted from the raw data set along radial lines at several different angles. These profiles are shown in Figure 13. The interpretation of four-dimensional data presented as profile sections from various angles on a 2-D graph, all at a common specimen crack process state (the fourth "dimension" or parameter) can be a bit difficult to comprehend.

To simplify the interpretation, first consider that all information on the graph is from a common state in the specimen testing process. Visualize, in this case, the physical specimen has been loaded until the CMOD reached $3.8 \mathrm{~mm}$, and the specimen was physically sectioned to reveal the crack profile at various positions within the specimen interior. All data in the graph represents this state as derived from the post-test microtopography data. Now, referring to Figure 4, consider 3 radial lines emanating from the coordinate origin, at angles of 18,32 , and $90 \mathrm{deg}$. The profiles shown on the plot of Figure 13 correspond to the surface heights along those lines. The abscissa, S, represents the distance from the origin along those lines.

The figure legend indicates the "local" state of the crack tip at each radial angular location. For the $90 \mathrm{deg}$ section, $\mathrm{S}=5.7 \mathrm{~mm}$ corresponds to the location of the back surface. Observing the upper and lower 90 deg profiles (long dash lines), one sees the transition from the high angle of tip stretching (blunting) transition to a much lower angle of tearing at $\mathrm{S}$ of about $5.1 \mathrm{~mm}$. Data processing

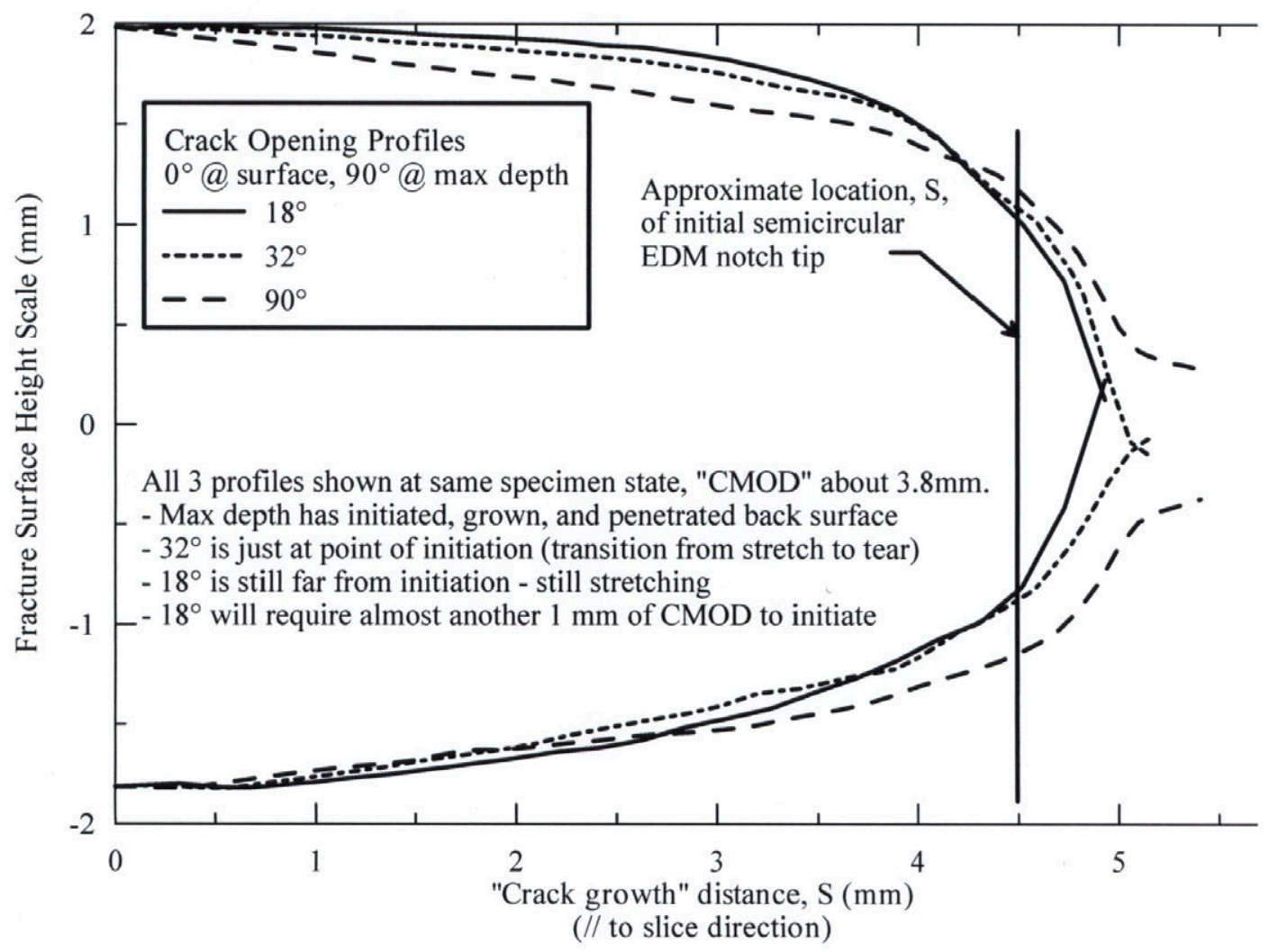

Figure 13. Crack opening profiles along radial lines originating at the origin for Specimen \#7. 
eliminated the last $0.2 \mathrm{~mm}$ of data on these lines. However, extrapolation along the latter portion of the profiles (e.g. 5.2 to $5.5 \mathrm{~mm}$ ) show that both upper and lower surface profiles intersect the back surface location (at $\mathrm{S}=5.7 \mathrm{~mm}$ ) before intersecting each other, i.e. the crack has penetrated the back surface at this state. Now observing the $32 \mathrm{deg}$ profiles (short dash lines), it can be seen that the local angles are just beginning the transition from high to low - crack initiation by definition. So, at a CMOD of $3.8 \mathrm{~mm}$, the crack is just initiating at the radial angular position of $32 \mathrm{deg}$. This particular state was chosen to show this feature. One can also estimate the local initiation CTOD, measured at the original EDM notch tip. In this case, at the radial location of $32 \mathrm{deg}, \mathrm{CTOD}_{\mathrm{i}}$ is about $2.0 \mathrm{~mm}$. By observing different locations at various states, the local initiation CTODs around the crack perimeter can be determined. Turning attention now to the $18 \mathrm{deg}$ section profile (near to the front surface, solid lines), we see continuing high angle at the leading portion of the profile. Additional data has been removed from this plot to eliminate confusion, but analyzing later specimen states show that the crack front at 18 deg does not transition to tearing growth until CMOD increases another $1 \mathrm{~mm}$ beyond the state shown in Figure 13.

\section{Research Plans \& Requirements for Microtopographic Analysis}

The information provided in the previous sections give a general overview of the state of microtopography analysis capabilities at INEEL, at the present state of knowledge and analysis development. The capability of extracting significant information about the fracture process from within the specimen interior of two quite different crack geometries in one of the materials of interest to this research project were demonstrated. A few areas where there is uncertainty in how to approach the data extraction, or where known process effects could be affecting the extracted information in unknown ways were identified.

A reliable method for assessment of crack growth at the maximum initial crack depth location has been demonstrated. The initiation CTOD and subsequent CTOA as a function of crack extension can be determined at this location with reasonable (not explicitly quantified) accuracy. Specimen geometric symmetry offers some benefit here, eliminating some of the three-dimension variability encountered at other positions around the initial crack perimeter. Once the crack has transitioned to a through-thickness crack growing along the plate width in a somewhat steady state, accurate estimates of the CTOA and crack front shape (tunneling development) can be determined. However, there are some factors related to the early stages of the fracture process that may need to be addressed by further research, if the principal investigators are not satisfied with the current estimated levels of accuracy. This is primarily associated with the region around the intersection of the initial crack (or notch) and the front surface.

\section{Plastic Flow Emanating From the Crack Tip and Proceeding To the Crack Flanks}

One issue needing further attention is that of plastic flow of material from the notch tip along curved slip lines that reconnect with the crack flanks within the specimen interior. Some theoretical problems (in the pure sense) are raised related to the basic premise of microtopographic analysis. That is, that once newly created fracture surface exits the near-tip process zone, it does not experience any further plastic (permanent) deformation. For some classes of materials, and certain crack geometries, McClintock's work has shown a relatively small effect on the extracted crack process parameters. However, this effect is substantial in some cases. For the two specimens investigated for this report, the effect is definitely observable. The fracture surface region where the initial planar EDM starter notches were, have measurable curvature. Plastic flow into this region has obviously occurred. However, this behavior may not be an insurmountable problem relative to microtopographic analysis. 
During initial crack tip blunting, plastic flow is confined to the near tip region. Material slip occurs on curved paths that emanate from the tip and intersect the crack flanks behind the tip, creating the rounded tip profile. As blunting increases, the intersection locations of the flow lines (surfaces) with the crack flanks move to points further behind the notch tip. At some point in the process, the flow field transitions from curved paths that intersect the crack flanks, to nominally straight paths that start at the process zone and angle forward to intersect the "back" surface. Our observations of plate-type specimens loaded in tension suggest this transition coincides with the transition from tip blunting to tearing crack growth. Rigorous experimental or analytical data are not available to support this hypothesis, but observations of many specimens analyzed suggest that this plastic flow from the crack tip region back to the crack flanks occurs primarily, if not entirely, during the initial blunting (stretching) of the crack tip.

This hypothesis supports the accuracy of the microtopographic analysis method from the point of crack growth initiation onward. Microtopography relies on the presumption that crack surfaces behind the near tip process zone - created by the fracture process do not undergo significant deformation after they exit the process zone, but only move by rigid rotation and translation relative to each other. The hypothesis presented, if proven, supports this presumption, i.e. that all significant fracture surface deformation is confined to the region behind the initial crack tip, and this deformation stops once the crack begins to grow in the tearing mode.

Following this reasoning, microtopography cannot accurately reproduce the deformation history of the precrack surfaces before initiation (as has been stated before), but can accurately assess the entire fracture process from the point of initiation to any time afterwards.

Following the transition from tip blunting to ductile tearing, the portion of the "rigid wedge" of material (as in non-hardening material slip line theory, see McClintock, [3]) that intersects the back surface is observed between the intersection of the two flow lines (more precisely, flow surfaces) with the back surface. As the crack tip advances toward the back surface, with the slip angle (between growth direction and slip lines) remaining nearly constant, the free surface of the rigid region is observed to decrease in height, reaching zero at the point of the crack penetrating through the specimen thickness.

This theory may be provable from an incompressibility standpoint - material lost to the crack opening void space creation (what we measure with microtopography) must be transported via flow along slip lines (surfaces in three dimensional cases) to a free surface somewhere else in the specimen. Flow from the crack tip perpendicular to the crack growth direction would not be a stable state in the PTC specimen case (the flow lines would effectively extend to infinity). Therefore, it seems likely the flow field "flips" from the "behind the tip" flow path (during blunting) to the "back surface" flow path (during tearing) rather abruptly. Experimental observations support this type of behavior.

\section{$(\mathrm{X}, \mathrm{Y})$ Positional Transformation of Fracture Surface during Crack Growth}

The spatial transformation of fracture surface positions during the ductile fracture process was touched on earlier. Figure 1 and Figure 2 show the rather severe distortion of the boundary of the fracture surface in its final state. Recall both specimens originally had a rectangular boundary of the fracture "plane." Front and back surfaces were planar (straight lines in the views of Figure 1 and Figure 2). One positive aspect of the issue is that in the absence of significant Mode II or Mode III deformation, the coordinates of the upper and lower fracture surface points transform in an identical fashion. In other words, a material point on the lower fracture surface at $P_{1}=\left(x_{i}, y_{i}\right)$ in the final state still corresponds to the material from which it separated on the upper surface at $P_{u}=\left(x_{i}, y_{i}\right)$. The same transform function, presently unknown, applies to points on both final surfaces in identical 
fashion. One can appreciate that this allows the analyses based on separation of the two surfaces to remain valid for small increments around any given location. However, there are analyses affected to various (unknown) extents or amounts by this spatial transform that may prove beneficial in the overall understanding of the ductile fracture process.

For example, consider the instantaneous crack border location analysis. Given the coordinate system of the final fracture surface state, $\mathrm{X}_{\mathrm{f}}$, a contour of constant separation on those surfaces does describe the locus of material points that were the instantaneous crack front at some state. However, the shape of that isoline generated by microtopographic analysis is not necessarily representative of the true crack front shape as it existed during the fracture process. This is one case where the coordinate transform function is required, to transform the final coordinates, $\mathrm{X}_{\mathrm{f}}$, to the desired state coordinates, $\mathrm{X}_{\mathrm{i}}$. Such a transform function will allow accurate representation of instantaneous crack border shape at any point in the fracture process. While this is not an immediate need in the early stages of the proposed research, it may be required when the effort turns to modeling the transition from dominant through-thickness growth to dominantly lateral growth of the through-thickness crack.

An experimental approach to derive the coordinate transform function for a given specimen material and geometry has been devised. In simple terms, the outer surface shape (contours in the nominal crack plane) will be measured in a nearly continuous manner during crack blunting and growth. These data will be correlated to some parameter, e.g. CMOD, that can be tied to the microtopography data analysis. At any given state of the analysis, we will have a geometric boundary condition for the transform function. By applying knowledge of incremental crack extension in combination with these boundary conditions, a coordinate transform function can be developed to incrementally reposition the interior fracture surface material points for consecutive fracture process states.

By developing this solution, we create the capability of providing a complete, three-dimensional, kinematic description of the entire fracture process occurring throughout the entire fracture zone in any individual test specimen. Then, the ductile fracture process for any ductile material with any desired initial crack geometry can be fully described in kinematical terms, with high levels of confidence in the extracted parameters.

\section{REFERENCES}

1. Lloyd, W. R., "Microtopography for ductile fracture process characterization - Part I: Theory and methodology," Engineering Fracture Mechanics 70, pp. 387-401, 2003.

2. Lloyd, W. R., and McClintock, F. A., "Microtopography for ductile fracture process characterization - Part II: Application for CTOA analysis," Engineering Fracture Mechanics 70, pp. 403-415, 2003.

3. McClintock, F. A., "Plasticity Aspects in Fracture," in Fracture: An Advanced Treatise, vol. 3, Liebowitz, H., ed., Academic Press, New York, 1971. 